\title{
Pluriharmonic maps into Kähler symmetric spaces and Sym's formula
}

\section{J.-H. Eschenburg • P. Quast}

Published online: 5 August 2009

(C) Springer-Verlag 2009

\section{Erratum to: Math. Z. DOI 10.1007/s00209-008-0472-9}

Let $M$ be a simply connected Riemann surface and $S^{2}$ the round 2-sphere. A key observation in our paper [4] is a generalization of the following simple fact:

Lemma A smooth map $h: M \rightarrow S^{2}$ is harmonic iff the $\mathbb{R}^{3}$-valued one-form $\gamma=\left(h \times h_{y}\right) \mathrm{d} x-$ $\left(h \times h_{x}\right) \mathrm{d} y$ is closed.

In Theorem 2.2 of our paper [4] we assign this observation to O. Bonnet [2], which is wrong. As explained by Hélein [5], it can be viewed as Noether's theorem for the variational problem for harmonic maps into the sphere, and it was used before in $[6,3,7]$ in order to study the corresponding evolution equation. It probably does not go back to the times of Bonnet. However, Bonnet [2] showed that all surfaces of constant mean curvature (those with harmonic Gauss map $h$ ) arise up to scaling as $f=g \pm h$ where $g$ is a surface of constant Gaussian curvature. But such surface $g$ is obtained by integrating the above one-form $\gamma$, (i.e. $\mathrm{d} g=\gamma$ ), see Equation (27) in our paper [4]. Therefore, we called $\gamma$ Bonnet form and $g$ Bonnet-Sym-Bobenko map since Sym [8] and Bobenko [1] have constructed $g$ in a different way. This might be misleading, and we want to point out that there is no doubt about the priority of Sym and Bobenko regarding the construction of $g$.

\section{References}

1. Bobenko, A.: Constant mean curvature surfaces and integrable equations. Russ. Math. Surv. 46, 1-45 (1991)

The online version of the original article can be found under doi:10.1007/s00209-008-0472-9.

J.-H. Eschenburg $(\bowtie) \cdot$ P. Quast

Institut für Mathematik, Universität Augsburg, 86135 Augsburg, Germany

e-mail: eschenburg@math.uni-augsburg.de

P. Quast

e-mail: peter.quast@math.uni-augsburg.de 
2. Bonnet, P.O.: Notes sur une propriété de maximum relative à la sphère. Nouvelles Annales de mathématiques XII, 433-438 (1853)

3. Chen, Y.M.: The weak solution to the evolution problem of harmonic maps. Math. Z. 201, 69-74 (1989)

4. Eschenburg, J.-H., Quast, P.: Pluriharmonic maps into Kähler symmetric spaces and Sym's formula. Math. Z. (2009). doi:10.1007/s00209-008-0472-9

5. Hélein, F.: Harmonic Maps, Conservation Laws and Moving Frames, 2nd edn. Cambridge University Press, Cambridge (2002)

6. Keller, J., Rubinstein, J., Sternberg, P.: Reaction-diffusion process and evoultion to harmonic maps. SIAM J. Appl. Math. 49(6), 1722-1733 (1989)

7. Shatah, J.: Weak solutions and developments of singularities of the $\mathrm{SU}(2) \sigma$-model. Commun. Pure Appl. Math. 41, 459-469 (1988)

8. Sym, A.: Soliton surfaces and their applications (Soliton geometry from spectral problems). In: Geometric Aspects of the Einstein Equations and Integrable Systems. Lecture Notes in Physics, vol. 239, pp. 154-231. Springer, Berlin (1986) 